\title{
Efeito do índice glicêmico no gasto energético e utilização de substrato energético antes e depois de exercício cicloergométrico
}

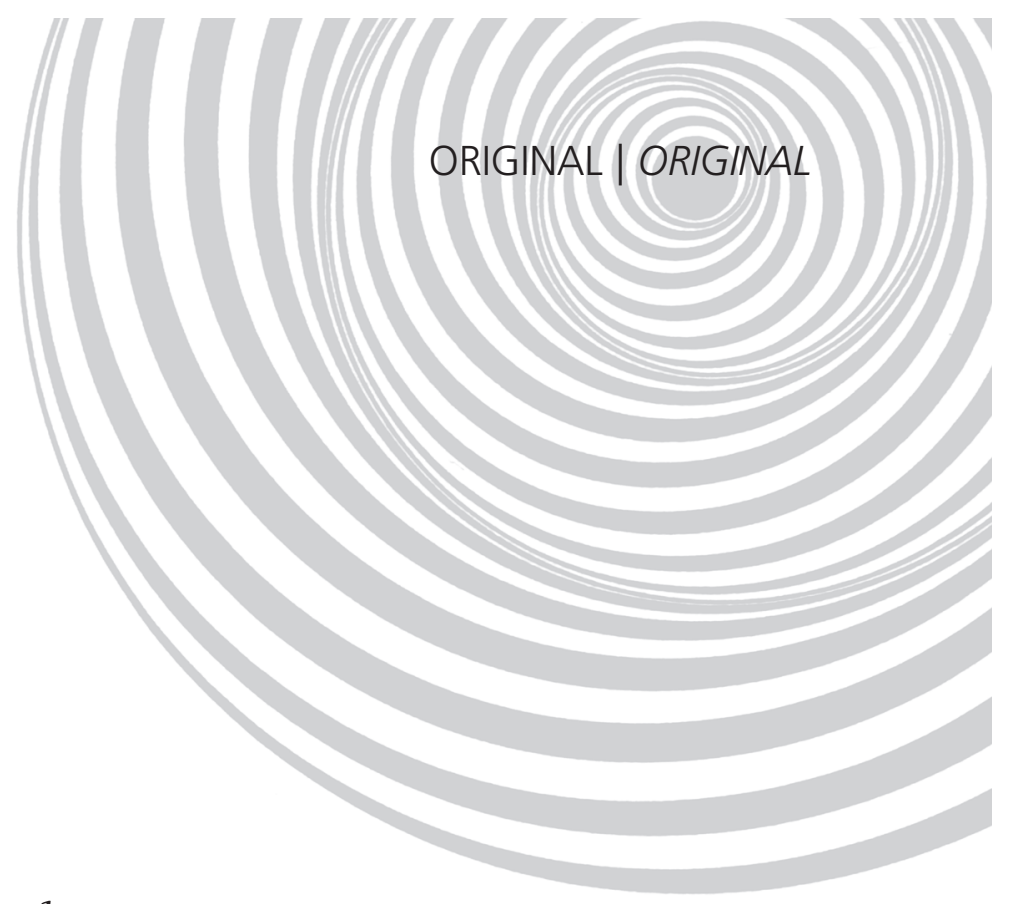

\section{Effect of glycemic index on energy expenditure \\ and energy substrate utilization before and \\ after exercise on a stationary bicycle}

\author{
Paula Guedes COCATE ${ }^{1}$ \\ Rita de Cássia Gonçalves ALFENAS ${ }^{1}$ \\ Letícia Gonçalves PEREIRA' \\ Josefina BRESSAN' ${ }^{1}$ \\ João Carlos Bouzas MARINS² \\ Paulo Roberto CECON ${ }^{3}$
}

RE S U M O

\section{Objetivo}

No presente artigo, avaliou-se o efeito do consumo, durante cinco dias consecutivos, de refeições diferindo em índice glicêmico no gasto energético, na oxidação de substrato energético e no consumo excessivo de oxigênio após o exercício.

\section{Métodos}

Participaram do estudo 15 homens bem treinados, com idade de $M=24,4, D P=3,70$ anos e consumo máximo de oxigênio $\left(\mathrm{VO}_{2 \max }\right)$ de $\mathrm{M}=70,00, \mathrm{DP}=5,32 \mathrm{~mL}(\mathrm{~kg} \cdot \mathrm{min})^{-1}$. Após o consumo das refeições, os participantes permaneceram por noventa minutos no calorímetro indireto Deltatrac ${ }^{\circledR}$, para a avaliação dos parâmetros metabólicos. A seguir, foi realizado um exercício de 85 a 95\% da frequência cardíaca máxima, em três estágios de dez minutos. Os parâmetros metabólicos foram novamente avaliados durante os sessenta minutos pós-exercício.

\section{Resultados}

Os tratamentos aplicados no estudo não afetaram o gasto energético, o consumo excessivo de oxigênio e a oxidação lipídica após o exercício. Entretanto, a taxa de oxidação de gordura foi maior durante os noventa

\footnotetext{
${ }^{1}$ Universidade Federal de Viçosa, Departamento de Nutrição e Saúde. Av. PH Rolfs s/n., Campus Universitário,36570 000,

Viçosa, MG, Brasil. Correspondência para/Correspondence to: PG. COCATE. E-mail: <guedescocate@yahoo.com.br>.

2 Universidade Federal de Viçosa, Departamento de Educação Física. Viçosa, MG, Brasil.

3 Universidade Federal de Viçosa, Departamento de Informática. Viçosa, MG, Brasil.
} 
948 | P.G. COCATE et al.

minutos no grupo que consumiu a refeição de alto índice glicêmico antes do exercício, em relação ao da refeição de baixo índice glicêmico. Além disso, a taxa de oxidação lipídica do período pós-prandial foi inferior àquela obtida no período pós-exercício.

\section{Conclusão}

Os resultados sugerem que enquanto o consumo de refeições de baixo índice glicêmico pode não exercer efeito benéfico, a realização de exercício físico pode promover maior oxidação lipídica e consequentemente afetar a redução do teor de gordura corporal.

Termos de indexação: Consumo de oxigênio. Exercício físico. Índice glicêmico. Metabolismo energético. Substratos.

\section{A B S T R A C T}

\section{Objective}

The present study assessed, on 5 consecutive days, the effect of consuming meals with different glycemic indices on energy expenditure, energy substrate oxidation and excessive oxygen consumption after exercise.

\section{Methods}

A total of 15 well trained men aged $M=24.4, S D=3.70$ years with a mean body mass index of $M=21.97$, $S D=1.46 \mathrm{~kg} / \mathrm{m}^{2}$ and maximum oxygen uptake $\left(\mathrm{VO}_{2 \mathrm{ma}}\right)$ of $M=70.00, S D=5.32 \mathrm{~mL}(\mathrm{~kg} . \mathrm{min})^{-1}$ participated in the study. After the meal, the participants remained 90 minutes in the indirect calorimeter Deltatrac ${ }^{\circledR}$ for assessment of the metabolic parameters. Next, they exercised at 85-95\% of their maximum heart rate in three bouts of 10 minutes. The metabolic parameters were reassessed within the 60 minutes following the exercise.

\section{Results}

The study treatments did not affect energy expenditure, excessive oxygen consumption or fat oxidation after exercise. However, the rate of fat oxidation in the 90 minutes that followed the meal was higher in those who consumed the high-glycemic index meal than in those who consumed the low-glycemic index meal. Moreover, the postprandial fat oxidation rate was lower than that observed after the exercise.

\section{Conclusion}

These results suggest that, while the consumption of low-glycemic index meals may not have beneficial effects, exercise can promote greater fat oxidation and, consequently, reduce body fat.

Indexing terms: Oxygen consumption. Exercise. Glycemic index. Energy metabolisme. Substrate.

\section{N T R O D U ÇÃ O}

A prevalência da obesidade vem aumentando consideravelmente no mundo, afetando todos os níveis sociais, sendo considerada atualmente uma epidemia mundial. Fatores ambientais como baixo nível de atividade física e alto consumo alimentar têm sido considerados como os principais intervenientes no aumento desta enfermidade. Sendo assim, a adoção de estratégias capazes de maximizar o gasto energético pós-prandial e a oxidação de gordura é importante no tratamento da obesidade?

Alguns autores têm reportado que o gasto energético após um exercício de alta intensidade é superior ao observado em outro de baixa inten- sidade ${ }^{2,3}$. Tem sido relatado também que, além de levarem ao aumento do consumo de oxigênio, os exercícios de alta intensidade favorecem o aumento da oxidação de gordura, efeito este benéfico para o controle de peso e do teor de gordura corporal ${ }^{4}$.

Os resultados obtidos em alguns estudos sugerem que o consumo de uma refeição contendo alimentos de Baixo Índice Glicêmico (BIG) pode favorecer o aumento da oxidação de lipídeos durante a realização de exercício cicloergométrico e corridas, em relação aos alimentos de Alto Índice Glicêmico (AIG) ${ }^{5,6}$. O efeito do Índice Glicêmico (IG) na utilização de substratos energéticos no período pós-exercício tem sido pouco explorado na literatura. 
Em outro estudo, verificou-se ainda que o consumo de uma refeição contendo alimentos de BIG, três horas antes de uma corrida (intensidade de $70 \%$ do consumo máximo de oxigênio), proporcionou maior estabilidade glicêmica e insulinêmica, quando tal exercício foi realizado até a exaustão ${ }^{7}$. Esses resultados ${ }^{5-7}$ sugerem que, além de garantir a oferta de glicose em concentrações constantes para ser utilizada como substrato energético durante o exercício, o consumo de alimentos de BIG pode favorecer a redução da quantidade de gordura corporal.

Em pesquisa recente à base de dados MedLine, SciELO, Science Direct, PubMed, verificou-se que apenas um estudo ${ }^{8}$ avaliou a influência aguda da ingestão de refeições apresentando IG distintos na oxidação de substrato e no gasto energético, de uma a duas horas após o exercício. Destaca-se ainda que, na maioria dos estudos publicados sobre este assunto, foi avaliado o efeito do consumo de apenas uma refeição de AIG ou BIG no gasto energético e oxidação de substratos $^{5-7}$. Além disso, Brand-Miller \& Foster-Powell ${ }^{9}$ recomendam que o consumo de duas refeições diárias de BIG seja capaz de promover efeitos benéficos à saúde.

Assim, esse estudo objetivou averiguar o efeito do consumo, durante cinco dias consecutivos, de duas refeições de AIG ou de BIG, no gasto energético, na oxidação de carboidrato e gordura antes e após exercício cicloergométrico de alta intensidade, bem como a influência destes tratamentos no consumo excessivo de oxigênio após o exercício.

\section{MÉ T O D O S}

Participaram do presente estudo 15 homens, com idade de Média $(M)=24,4$, Desvio-Padrão (DP)=3,7 anos, índice de massa corporal (IMC) de $M=21,97, D P=1,46 \mathrm{~kg}$, percentual de gordura $M=7,93, D P=2,36 \%$, consumo máximo de oxigênio $\left(\mathrm{VO}_{2 \max }\right)$ de $\mathrm{M}=70,00, \mathrm{DP}=5,32 \mathrm{~mL}$ (kg.min) $)^{-1}$. Tais participantes não estavam em dieta para controle de peso; apresentavam peso estável (alteração<3kg) nos últimos três meses; apresentavam excelente nível de aptidão física, de acordo com a classificação proposta por Cooper $^{10}$; não obtiveram resposta positiva a todas as perguntas do questionário "Par Q \& Você"11; apresentavam pressão arterial normal em repouso, segundo os critérios da Sociedade Brasileiras de Hipertensão ${ }^{12}$; eram praticantes regulares (no mínimo três vezes por semana) de atividade física; não eram fumantes; não eram usuários de álcool ou de medicamentos que afetam a ingestão de alimentos ou o metabolismo energético.

\section{Desenho experimental}

Trata-se de um estudo em crossover, em que os voluntários foram designados aleatoriamente a um dos dois grupos experimentais e consumiram refeições de AIG ou BIG no desjejum e no lanche da tarde. As refeições testadas foram ingeridas em laboratório durante cinco dias consecutivos. O desjejum foi ingerido até o quinto dia e o lanche da tarde, até o quarto dia de cada etapa. Houve um intervalo de pelo menos uma semana entre as etapas experimentais. As demais refeições do dia foram ingeridas em condições de vida livre. Os voluntários receberam uma lista discriminando os alimentos quanto ao $\mathrm{IG}^{13} \mathrm{e}$ foram orientados a ingerir preferencialmente, alimentos de AIG ou BIG, de acordo com a etapa em que estavam participando.

No primeiro e no último dia de cada etapa do estudo, os voluntários se apresentaram ao laboratório, após 12 horas de jejum, para avaliação da composição corporal, utilizando a balança TANITA, modelo TBF-300A ${ }^{\circledR}$. Também foi realizada avaliação antropométrica, do gasto energético de repouso, da taxa de oxidação lipídica e de carboidrato, e do quociente respiratório. Após o consumo de refeições de AIG ou de BIG, os mesmos parâmetros metabólicos foram reavaliados. A seguir, os voluntários realizaram um exercício cicloergométrico. No período pós-exercício as variáveis metabólicas citadas foram novamente avaliadas. O experimento foi conduzido sob tem- 
peratura ambiente laboratorial entre 24 e $26^{\circ} \mathrm{C}$ e umidade relativa do ar entre 70 e $75 \%$.

Após a realização de todos os testes, os voluntários foram liberados para exercer suas atividades normais em condições de vida livre, mantendo o registro dos tipos e quantidades de alimentos ingeridos do primeiro ao quinto dia do estudo. Durante o experimento, os participantes foram solicitados a manter o mesmo nível de atividade física (Figura 1).

\section{Avaliação antropométrica e da composição corporal}

Foi calculado o $\mathrm{IMC}^{14}$, relacionando o peso $(\mathrm{kg})$ e a altura (metros ao quadrado). Os voluntários foram pesados utilizando-se balança eletrônica digital da marca Tanita ${ }^{\circledR}$, com capacidade de $150 \mathrm{~kg}$, graduação de peso de 0,1 kg e graduação de gordura de 0,1\%, usando o mínimo de roupa possível. A altura foi determinada utilizando-se um antropômetro vertical milimetrado, com extensão de $2 \mathrm{~m}$ e escala de 0,5cm. Para a determinação do peso e da altura, os avaliados encontraram-se de pé, em posição firme, com os braços relaxados e cabeça no plano horizontal. Foram considerados eutróficos os voluntários que apresentaram valores de IMC ${ }^{15}$ de 18,5 a 24,9 kg/m².
A composição corporal (quantidades e percentuais de gordura corporal total, massa magra e água corporal total) dos participantes do estudo foi avaliada utilizando-se o método da bioimpedância bipolar (TANITA ${ }^{\circledR}$, modelo TBF-300A). Esse equipamento contém sensores elétricos em locais específicos na plataforma de pesagem, os quais ao entrarem em contato direto com a sola do pé do analisado, promovem a mensuração da composição corporal (gordura corporal e massa livre de gordura) em função da dificuldade de condução de corrente elétrica pelo corpo. Para tal mensuração, os voluntários foram orientados a fazer o mínimo de atividade física e se abster do consumo de café no dia anterior ao teste, não consumir álcool nas 48 horas antecedentes e evitar a ingestão de água nas horas precedentes ao teste.

\section{Refeições testadas}

A refeição de BIG era composta de iogurte de morango desnatado, cereal matinal à base de trigo (All Bran da Kellogg's ${ }^{\circledR}$ ), suco de uva concentrado, frutose em pó, pão de forma multigrãos, margarina e maçã. A refeição de AIG continha bebida esportiva, leite integral, glicose em pó, cereal matinal de milho (Corn Flakes da Kellogg's $\left.{ }^{\circledR}\right)$, pão de forma branco, margarina e

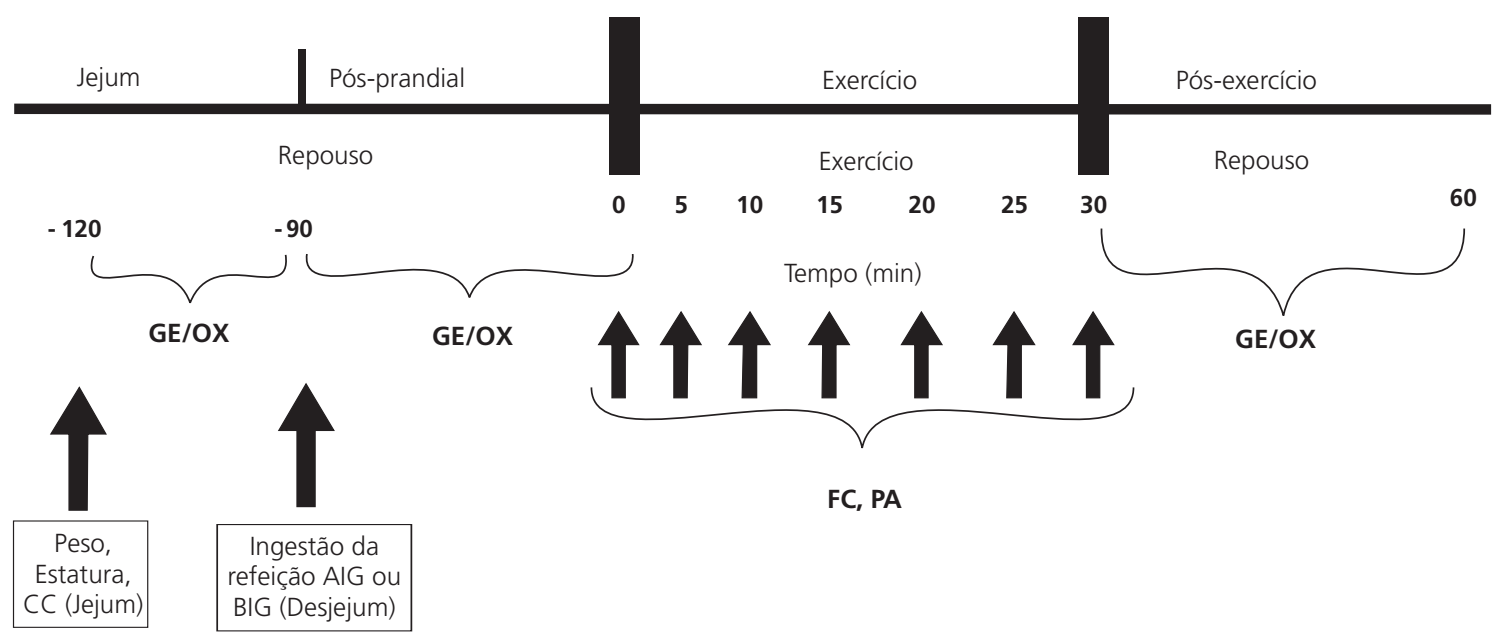

Figura 1. Representação esquemática do protocolo adotado no primeiro e no quinto dia das etapas experimentais do presente estudo. Viçosa (MG), 2007.

Nota: CC: composição corporal; AIG: alto índice glicêmico; BIG: baixo índice glicêmico; GE: gasto energético; QR:quociente respiratório; OX: avaliação da oxidação de gordura e carboidrato; FC: frequência cardíaca; PA: pressão arterial. 
fibra alimentar solúvel (Benefiber ${ }^{\circledR}$ ). A adição do suplemento de fibra solúvel à refeição de AIG foi feita com o intuito de igualar o teor de fibras desta refeição ao daquela de BIG. Desta forma, as refeições testadas apresentaram densidade calórica, composição de macronutrientes e de fibras totais semelhantes (Tabela 1).

Apesar da fonte de fibra predominante na refeição de BIG ter sido insolúvel e na de AIG ter sido solúvel, esta diferença quanto ao tipo de fibra não afetou o IG das refeições (mensurado em laboratório, conforme descrito no item "Determinação do índice glicêmico das refeições testadas", apresentado abaixo). Segundo alguns autores a redução da glicemia só é observada após o consumo de quantidades superiores ou iguais a $50 \mathrm{~g}$ de fibras solúveis por dia $^{16}$ ou quando essas fibras são ingeridas por indivíduos diabéticos ${ }^{17}$, situações não testadas nesse estudo.

Os participantes ingeriram todos os alimentos fornecidos dentro de 15 minutos. As refeições testadas apresentavam um aporte calórico equivalente a $1 / 3$ da taxa metabólica de repouso e aproximadamente $2 \mathrm{~g}$ de carboidrato disponível por quilo de peso corporal de cada participante ${ }^{18}$.

\section{Determinação do índice glicêmico das refeições testadas}

A seleção prévia dos alimentos de AIG e de BIG a serem incluídos nas refeições testadas foi feita baseando-se nos valores de IG publicados na Tabela Internacional de Índice Glicêmico ${ }^{13}$. A determinação do IG de tais refeições foi feita em um estudo piloto. Para tal, foram recrutados sete voluntários (dois homens e cinco mulheres) eutróficos $M=21,4, D P=2,5 \mathrm{~kg} / \mathrm{m}^{2}$ ), com idade de $\mathrm{M}=22,8, \mathrm{DP}=3,1$ anos, apresentando glicemia normal, não diabéticos, sem história familiar de diabetes ou de intolerância à glicose e sem uso de medicamentos que afetam a glicemia. Os voluntários ingeriram as refeições testadas uma vez. A glicose foi utilizada como alimento de referência, sendo ingerida três vezes por cada voluntário ${ }^{19}$.

Tais voluntários se apresentaram ao laboratório após 10-12 horas de jejum. Uma porção da refeição testada, contendo $50 \mathrm{~g}$ de carboidrato disponível, foi ingerida em 15 minutos. Alterações glicêmicas resultantes desta ingestão foram avaliadas pela determinação da glicemia capilar utilizando o aparelho One Touch U/tra ${ }^{\circledR}$, nos tempos 0 (imediatamente antes da ingestão), 15, 30, 45, 60, 90 e 120 minutos (após início da ingestão) ${ }^{19}$. A área positiva formada abaixo da curva de resposta glicêmica foi calculada pelo método trapezoida ${ }^{20}$. Os valores de IG das refeições foram calculados a partir da área obtida após a ingestão de cada refeição, sendo expressa em termos da porcentagem da resposta glicêmica obtida após a ingestão da glicose. O IG de cada refeição foi determinado a partir da média aritmética dos valores obtidos pelos sete voluntários ${ }^{19,20}$.

\section{Exercício físico}

Os voluntários realizaram um exercício cicloergométrico, com intensidade de 85 a 95\% da frequência cardíaca máxima, em três estágios com duração de dez minutos e intervalo de dois minutos. No período de repouso (imediatamente antes do exercício) e no quinto e décimo minuto de cada estágio, a frequência cardíaca foi monitorizada adotando um frequencímetro modelo M31 da marca Polar ${ }^{\circledR}$, sendo a pressão arterial

Tabela 1. Índice glicêmico, densidade energética, teor de macronutrientes e de fibras apresentados pelas refeições de alto índice glicêmico (AIG) e baixo índice glicêmico (BIG), utilizadas neste estudo. Viçosa (MG), 2007.

\begin{tabular}{|c|c|c|c|c|c|c|c|c|}
\hline \multirow{2}{*}{$\begin{array}{l}\text { Refeição } \\
\text { testada }\end{array}$} & \multirow{2}{*}{$\begin{array}{c}\text { Índice } \\
\text { glicêmico }\end{array}$} & \multirow{2}{*}{$\begin{array}{c}\text { Densidade } \\
\text { energética }(\mathrm{kcal} / \mathrm{g})\end{array}$} & \multicolumn{3}{|c|}{ (\% total kcal) } & \multicolumn{3}{|c|}{ Fibra (g/100/g) } \\
\hline & & & Carboidrato & Proteína & Gordura & Insolúvel & Solúvel & Total \\
\hline AIG & 79 & 1,4 & 83,5 & 7,2 & 9,4 & 1,4 & 13,4 & 14,8 \\
\hline BIG & 28 & 1,1 & 83,5 & 7,2 & 9,4 & 11,4 & 3,2 & 14,6 \\
\hline
\end{tabular}

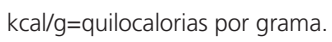


sistólica e diastólica mensurada por meio de um esfigmomanômetro e um estetoscópio da marca Wan Med $^{\circledR}$. Durante o exercício, os participantes consumiram $3 \mathrm{~mL}$ de água por quilo de peso corporal em três situações: após o aquecimento, aos cinco minutos do segundo estágio e no último minuto do último estágio.

\section{Determinação da frequência cardíaca máxima dos participantes}

Na semana anterior ao início do experimento, os participantes foram submetidos ao teste máximo de Balke, utilizando um cicloergômetro eletromagnético, da marca Ergo Fit ${ }^{\circledR}$, modelo Ergo Cicle 167. Neste teste, foram empregados estágios múltiplos, iniciando com carga de cinquenta Watts, a qual foi aumentada a cada dois minutos, com magnitude de cinquenta Watts, até 0 alcance do limite máximo ${ }^{21}$. A seguir, foi registrado o valor da última carga completada pelo voluntário em Watts, bem como o valor do peso corporal avaliado antes da realização do teste, para a determinação do $\mathrm{VO}_{2 \text { máx }}$ e do nível de aptidão física dos voluntários ${ }^{10}$. A intensidade do exercício foi determinada utilizando o valor da frequência máxima obtida no teste para o cálculo da frequência cardíaca de treino (FCT), que leva em consideração a frequência cardíaca (FC) de reserva, utilizando a seguinte fórmula: $\mathrm{FCT}=$ $F C_{\text {repouso }}+\%\left(\mathrm{FC}_{\text {máxima calculada }}-\mathrm{FC}_{\text {repouso }}\right)^{22}$.

\section{Avaliação do gasto energético e da oxidação de substrato energético}

Ao chegarem ao laboratório, os voluntários permaneceram em repouso por trinta minutos em ambiente silencioso, com pouca iluminação e com temperatura confortável, para evitar alterações causadas por frio ou ansiedade ${ }^{23}$. A seguir, procedeu-se a mensuração do metabolismo referente ao período de jejum, utilizando o aparelho de calorimetria indireta (Deltatrac $\|^{\circledast}$ Datex, Finlândia). Durante a mensuração, os voluntários foram man- tidos em repouso durante trinta minutos. Foram determinados os valores do Quociente Respiratório $(\mathrm{QR})$, relacionando os moles de gás carbônico $\left(\mathrm{CO}_{2}\right)$ expirado/moles de oxigênio $\left(\mathrm{O}_{2}\right)$ consumido. Este valor foi convertido em quilocalorias de calor produzido por metro quadrado de superfície cor-poral por hora, sendo determinado a seguir o gasto energético diário total ${ }^{24}$. No dia anterior à aferição do metabolismo energético, os voluntários foram orientados a evitar a ingestão de álcool e o excesso de atividade física.

Após esse período, os participantes ingeriram a refeição de AIG ou de BIG e retornaram ao aparelho de calorimetria indireta, permanecendo neste durante noventa minutos para mensuração do gasto energético, consumo de oxigênio $\left(\mathrm{VO}_{2}\right)$, produção de gás carbônico $\left(\mathrm{VCO}_{2}\right)$ e QR (período pré-exercício). Em seguida, os participantes fizeram um exercício cicloergométrico (intervalado, com três estágios de dez minutos de duração), com intensidade de 85 a 95\% da frequência cardíaca máxima, obtida no teste máximo de Balke, realizado na semana anterior.

Após a realização do exercício intervalado, os voluntários retornaram novamente ao calorímetro indireto, para a mensuração do gasto energético, durante sessenta minutos (período pós-exercício). O aumento do consumo de oxigênio pós-exercício em relação ao período de jejum (EPOC) foi calculado pela diferença entre o valor médio do $\mathrm{VO}_{2}$ obtido durante os sessenta minutos pós-exercício e o valor médio de $\mathrm{VO}_{2}$ obtido durante os trinta minutos em estado de jejum ${ }^{25}$.

As taxas de oxidação de lipídeos e carboidratos foram estimadas a partir do $\mathrm{VO}_{2}$ e VCO avaliados no período de determinação do metabolismo de repouso, no período pré e pós-exercício, usando as seguintes equações estequiométricas $^{26}$ :

Taxa de oxidação de lipídeos $(\mathrm{g} / \mathrm{min})=$ $1,695 \times \mathrm{VO}_{2}-1,701 \times \mathrm{VCO}_{2}$

Taxa de oxidação de carboidrato $(\mathrm{g} / \mathrm{min})=$ $4,585 \times \mathrm{VCO}_{2}-3,226 \times \mathrm{VO}_{2}$ 


\section{Análises estatísticas}

Para comparar o efeito dos tratamentos durante o estudo foi utilizado o teste de medidas repetidas de Análise de Variância (ANOVA) para as variáveis com distribuição normal e o Teste de Kruskal-Wallis para os que não apresentavam distribuição normal. Quando estes resultados se apresentaram significantes, utilizou-se o procedimento de comparações múltiplas de Tukey e Dunn's, respectivamente, para completá-los. As análises foram conduzidas utilizando o programa estatístico SigmaStat 3.0. Valores de probabilidade abaixo de 0,05 foram considerados estatisticamente significantes. Os resultados estão apresentados como médias e Desvio-Padrão (DP) quando se utilizou estatística paramétrica e mediana quando se utilizou estatística não-paramétrica.

O protocolo do presente estudo foi aprovado pelo Comitê de Ética em Pesquisas com Seres Humanos da Universidade Federal de Viçosa (MG), atendendo as orientações da resolução 196/96 do CNS, de 10/10/96, sobre experimentos com seres humanos (parecer $n^{\circ}$ 021/2006).

\section{RES U LTA D OS}

\section{Ingestão alimentar durante o estudo}

Apesar do consumo de carboidrato e proteína durante o estudo não ter diferido entre as etapas experimentais, verificou-se que a ingestão de lipídeos durante a etapa de BIG foi inferior $(p<0,05)$ à constatada na etapa de AIG. Entretanto, tal diferença não afetou a ingestão calórica dos participantes durante o estudo.

\section{Frequência cardíaca, pressão arterial sistólica e diastólica}

A frequência cardíaca e a pressão arterial apresentada pelos participantes não diferiram estatisticamente $(p>0,05)$ entre as etapas do estudo.

\section{Gasto energético, quociente respiratório e consumo de oxigênio}

a) Efeito do índice glicêmico das refeições: ao comparar o gasto energético e o QR entre o primeiro e quinto dia de cada etapa experimental do estudo, observou-se que ambos os parâmetros não foram modificados significativamente durante o estudo. Foi observado que o QR após o consumo da refeição de AIG foi significantemente menor do que de BIG, tanto no primeiro quanto no quinto dia de teste (Figura 2). Não foram constatadas diferenças no consumo de oxigênio no período de jejum, pós-prandial (antes do exercício) e pós-exercício entre os tratamentos adotados no estudo.

b) Efeito do exercício: verificou-se que o QR pós-prandial antes do exercício foi superior ao apresentado no pós-exercício em resposta ao consumo das refeições de $\mathrm{AIG}$ ou BIG, no mesmo dia de teste (Figura 2).

\section{Taxa de oxidação de substrato energético}

a) Efeito do índice glicêmico das refeições: as taxas de oxidação de gordura e de carboidrato durante o período de jejum foram semelhantes para os dois tipos de refeições testadas. A taxa de oxidação de carboidrato obtida no pós-prandial antes do exercício foi menor no primeiro e último dia de consumo de AIG do que de BIG (Figura 3).

Durante os noventa minutos pós-prandiais obteve-se uma taxa de oxidação de gordura maior, no primeiro e no último dia de consumo da refeição de AIG em relação àquela observada após a ingestão da refeição de BIG. Além disso, verificou-se que a taxa de oxidação lipídica obtida no período pós-prandial foi inferior àquela obtida no período pós-exercício, no primeiro e quinto dia de consumo de AIG e BIG (Figura 4).

b) Efeito do exercício:observou-se que a taxa de oxidação de carboidrato do período pós-prandial antes do exercício foi maior do que a obtida no período pós-exercício (Figura 3 ). Por 
954 | P.G. COCATE et al.

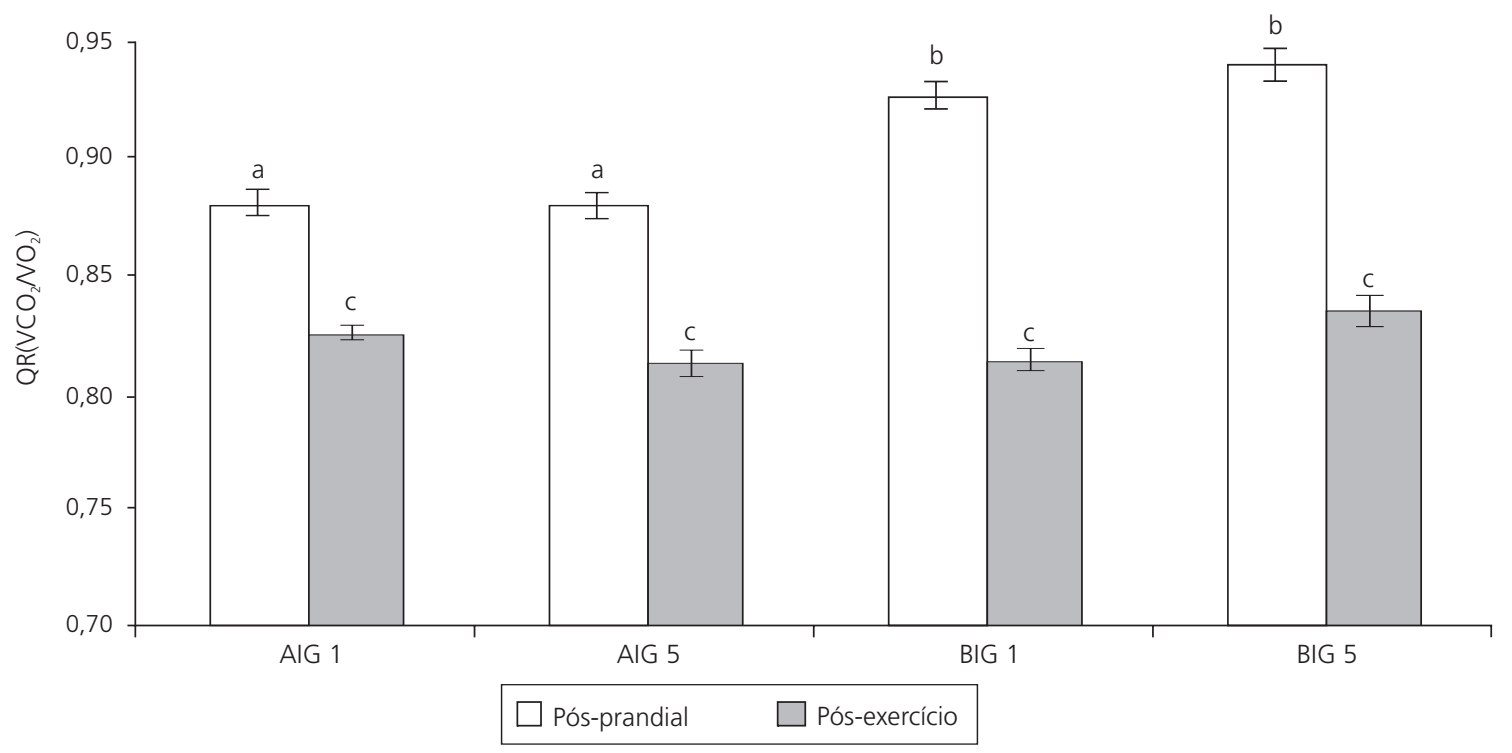

Figura 2. Mediana do quociente respiratório obtido no primeiro e no quinto dia antes e após a realização de exercício após o consumo da refeição. Viçosa (MG), 2007.

Nota: Barras acompanhadas de letras distintas diferem entre si. As diferenças encontradas foram constatadas pelo teste Kruskal-Wallis complementado pelo teste de Dunn's.

AIG1 e AIG5: alto índice glicêmico no primeiro e quinto dia, respectivamente; BIG1 e BIG5: baixo índice glicêmico no primeiro e quinto dia, respectivamente.

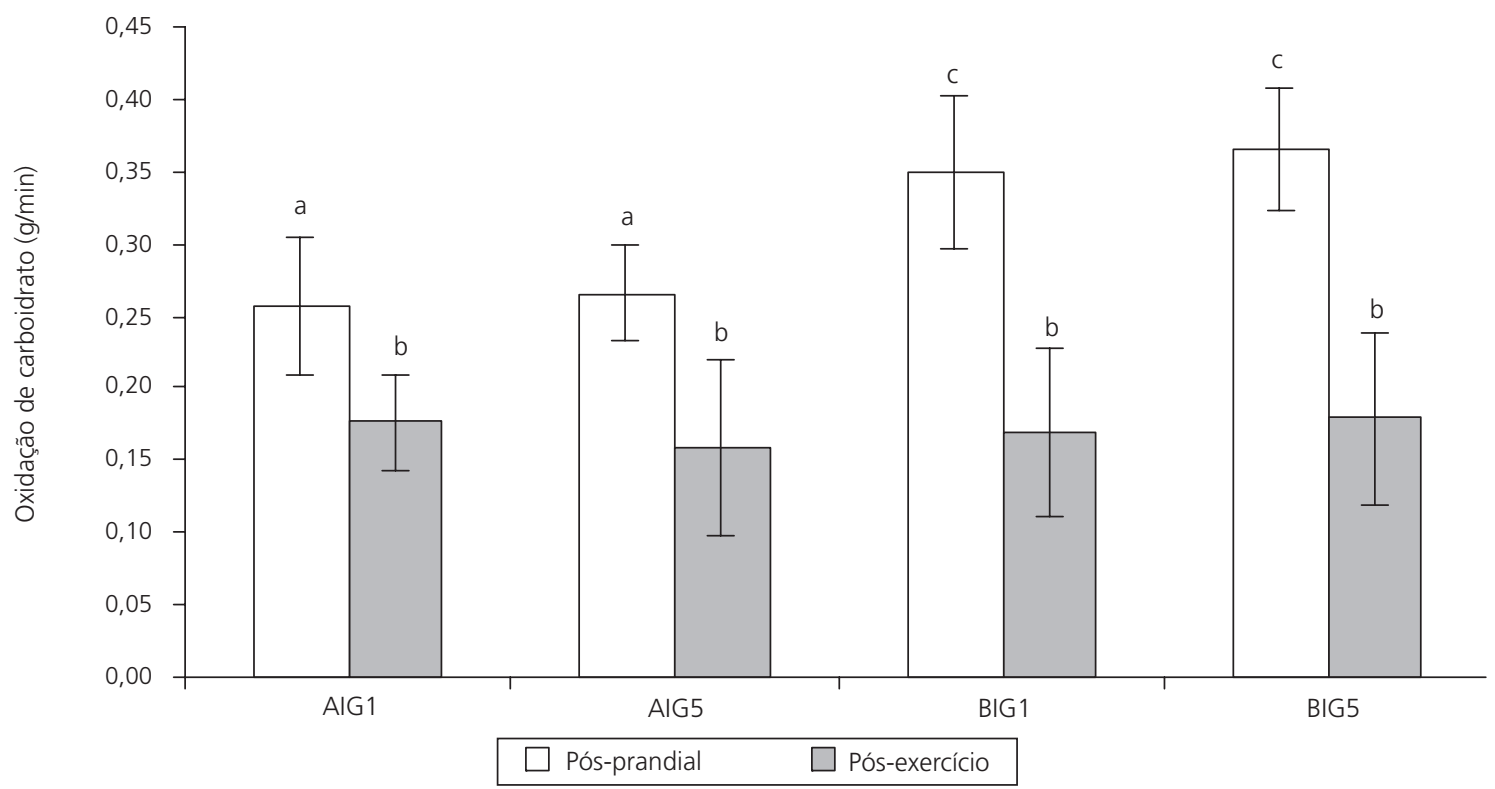

Figura 3. Médias e desvio-padrão da oxidação de carboidrato durante o período pós-prandial antes e após o exercício, no primeiro e no quinto dia de consumo da refeição. Viçosa (MG), 2007.

Nota: Barras acompanhadas de letras distintas diferem entre si. As diferenças foram constatadas pelo teste de Anova, complementado pelo teste de Tukey.

AIG1 e AIG5: alto índice glicêmico no primeiro e quinto dia, respectivamente; BIG1 e BIG2: baixo índice glicêmico no primeiro e quinto dia, respectivamente. 


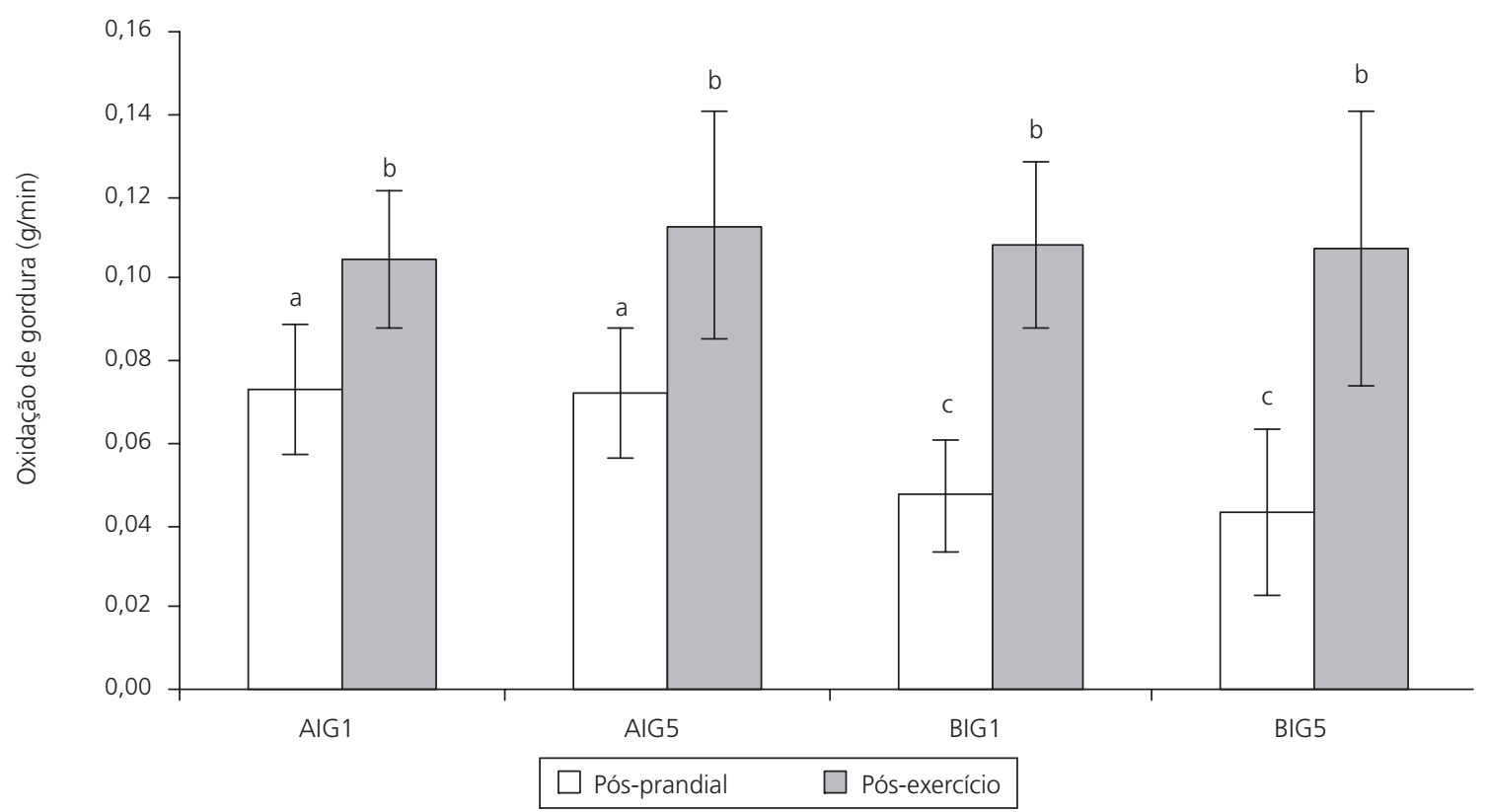

Figura 4. Média e desvio-padrão da oxidação de gordura durante o período pós-prandial antes e após exercício, no primeiro e no quinto dia de consumo da refeição. Viçosa (MG), 2007.

Nota: Barras acompanhadas de letras distintas diferem entre si. As diferenças encontradas foram constatadas pelo teste de Anova, complementado pelo teste de Tukey.

AIG1 e AIG5: alto índice glicêmico no primeiro e quinto dia, respectivamente; BIG1 e BIG5: baixo índice glicêmico no primeiro e quinto dia, respectivamente.

outro lado, a taxa de oxidação de gordura foi inferior no período pós-prandial antes do exercício em relação ao período pós-exercício em ambos os tratamentos (Figura 4).

\section{Consumo excessivo de oxigênio após o exercício (EPOC)}

Não foi constatada diferença do consumo excessivo de oxigênio após o exercício (EPOC) entre os tratamentos. Os valores obtidos no primeiro e quinto dia em que os participantes consumiram a refeição de AIG foram de $M=3,72$, $\mathrm{DP}=1,85 \mathrm{~L} / \mathrm{min}$ e $\mathrm{M}=3,67, \mathrm{DP}=1,77 \mathrm{~L} / \mathrm{min}$, respectivamente. Já para o primeiro e último dia de consumo da refeição de BIG, os valores obtidos corresponderam a $\mathrm{M}=3,57, \mathrm{DP}=2,34 \mathrm{~L} / \mathrm{min}$ e $\mathrm{M}=3,80$, $\mathrm{DP}=1,49 \mathrm{~L} / \mathrm{min}$, respectivamente.
DISCUSSÃ O

Nesse estudo, o gasto energético avaliado em jejum (taxa metabólica de repouso), no período pós-prandial e no pós-exercício não foi afetado pelo IG das refeições testadas ou entre o primeiro e quinto dia de cada etapa experimental. Resultado semelhante foi constatado no estudo de Abete et al..$^{27}$ em que o consumo de dietas de AIG e a de BIG durante oito semanas não afetou a taxa metabólica de repouso. Em outro estudo ${ }^{6}$ em que se avaliou o efeito agudo do IG, o consumo de refeições de AIG e BIG também não afetou o gasto energético pós-prandial (pré-exercício). Os resultados desses estudos sugerem que o gasto energético não seja afetado em função do IG apresentado pelos alimentos.

Apesar da ausência de diferença no gasto energético entre os tratamentos do presente estu- 
do, o QR atingido durante os noventa minutos após consumo das refeições de AIG e BIG (período pós-prandial antes do exercício) foi superior ao obtido no período pós-exercício. O valor do QR indica o tipo de macronutriente preferencialmente metabolizado. Enquanto a obtenção de valores próximos de 1,0 indica a ocorrência de maior oxidação de carboidrato, valores próximos a 0,7 indicam a oxidação preferencial de gordura ${ }^{23}$. O QR médio obtido durante os noventa minutos pós-prandiais variou entre 0,87 e 0,93 no primeiro e no quinto dia das duas etapas do estudo. Como esses valores estão mais próximos de 1,0 do que de 0,7 , eles indicam a ocorrência de oxidação preferencial de carboidrato. De acordo com Bennard \& Doucet ${ }^{8}$, após o consumo de refeições ricas em carboidrato, a glicose passa a ser o principal substrato oxidado no organismo. Assim, o resultado obtido no presente estudo reflete o alto teor de carboidratos $(83,5 \%)$ das refeições testadas.

A ingestão da refeição de BIG proporcionou maior $\mathrm{QR}$, maior oxidação de carboidrato e menor oxidação de gordura, durante os noventa minutos após o seu consumo do que após o consumo da refeição de AIG. Resposta semelhante foi constatada no período pós-exercício em outro estudo ${ }^{28}$, em que foi avaliado o efeito do consumo de $50 \mathrm{~g}$ de frutose (BIG) ou $50 \mathrm{~g}$ de glicose (AIG) na resposta metabólica. Entretanto, os resultados de outras pesquisas indicam que o consumo de dietas de BIG favorece o aumento da oxidação de lipídeos no período pós-prandial antes e durante o exercício em relação às dietas de AIG, apresentando concentrações de macronutrientes e densidade calórica semelhantes $5^{6,7,18}$.

A divergência nos resultados desses estudos pode ter ocorrido em função da adição de frutose em pó à refeição de $\mathrm{BIG}$, tanto no presente estudo quanto no de Tittelbach et al. ${ }^{28}$. A frutose é especialmente metabolizada no fígado. Sua rápida entrada no hepatócito é mediada pela GLUT 2, não havendo gasto de energia ou necessidade de atuação da insulina. No hepatócito, a frutose é rapidamente fosforilada no carbono 1 pela frutoquinase ou cetoquinase, ou no carbono 6 pela hexoquinase. A frutose-1 fosfato, por sua vez, é dividida em duas trioses, diidroxiacetona e gliceraldeído-fosfato, em uma reação mediada pela aldolase B. Essas duas trioses podem ser condensadas para formar a frutose-1,6-difosfato, a qual pode ser convertida em glicose. A seguir, a ocorrência de glicólise e a fosforilação oxidativa (oxidação de carboidrato) são estimuladas, promovendo um aumento do QR ${ }^{29}$.

A taxa de oxidação de gordura após a realização de exercício de alta intensidade tem sido associada ao estímulo do sistema nervoso simpático, resultando no aumento das concentrações de ácido graxo livre na corrente sanguínea, os quais são utilizados para a produção de energia $^{4,30}$. De acordo com Foureaux et al. ${ }^{30}$, há um aumento de 20 a 35\% da ocorrência de lipólise no adipócito após a realização de exercícios físicos. A maior taxa de oxidação de gorduras associada ao maior gasto energético obtido após a realização de exercícios de alta intensidade ${ }^{3}$ pode favorecer a perda de peso corporal ${ }^{4}$.

Um outro estudo 4 envolveu a participação de oito homens moderadamente treinados. Após a realização de um exercício de baixa intensidade (38\% do $V_{2 \text { max' }}$ duração de $M=65, D P=9$ minutos), um de alta intensidade $\left(77 \%\right.$ do $\mathrm{VO}_{2 \text { max' }}$ duração de $\mathrm{M}=33, \mathrm{DP}=6$ minutos) e de um período de repouso ( $\mathrm{M}=67, \mathrm{DP}=11$ minutos de duração), os participantes consumiram uma refeição padrão de $900 \mathrm{kcal}$ (45\% de carboidrato, 36\% de lipídeo e $19 \%$ de proteína). A resposta metabólica foi avaliada de 60 a 270 minutos pós-exercício e após o repouso, correspondendo ao período pós-prandial imediato. Verificou-se que o consumo de oxigênio foi superior após o exercício de alta intensidade em relação à situação de exercício de baixa intensidade e condição de repouso. Destaca-se que o consumo de oxigênio apresenta relação direta com o gasto energético, ou seja, para cada litro de oxigênio consumido, são gastas aproximadamente $5 \mathrm{kcal}^{31}$. Assim, o maior consumo de oxigênio após a realização do exercício de alta intensidade no período pós-prandial imediato pode contribuir para que haja maior gasto energético, contribuindo assim para a perda de peso corporal ${ }^{30}$. 
Neste estudo, o EPOC avaliado durante os sessenta minutos pós-exercício foi de aproximadamente 3,68L após consumo das refeições testadas, correspondendo a um gasto energético extra de aproximadamente de $18,41 \mathrm{kcal} / \mathrm{min}$. Resultado semelhante foi obtido em um outro estudo $^{32}$ em que o EPOC ( $\left.M=3,24, D P=14,8 \mathrm{~L}\right)$ foi avaliado durante $M=40,3, D P=14,8$ minutos após um exercício cicloergométrico com duração de trinta minutos e intensidade de $70 \%$ do $\mathrm{VO}_{2 \max }$. Além disso, no estudo citado anteriormente ${ }^{32}$ verificou-se uma queda expressiva do $\mathrm{QR}$ no período pós-exercício em relação ao valor obtido em jejum. A queda do QR evidencia uma maior oxidação de gordura, contribuindo assim para a redução do teor de gordura corporal.

A realização de um exercício cicloergométrico durante trinta minutos com intensidade de $70 \%$ do $\mathrm{VO}_{2 \max }$ resultou em EPOC ainda mais elevado que o obtido nos estudos citados anteriormente $^{33}$. Segundo alguns autores ${ }^{32}$, dependendo do nível de condicionamento físico apresentado, a realização de exercício resulta em regulação metabólica, a qual afeta o EPOC obtido. Assim, quanto maior o condicionamento físico do indivíduo, mais rápida será a regulação metabólica apresentada pelo mesmo, evitando elevação muito acentuada do EPOC. Desta maneira, o maior condicionamento físico dos participantes do atual estudo e do estudo de Short \& Sedlock ${ }^{32}$ favoreceu a obtenção do EPOC mais baixo do que neste último estudo ${ }^{33}$ citado.

Vale ressaltar que após revisão nas bases de dados PubMed, SciELO e Science Direct não foi encontrado nenhum estudo que tenha avaliado o efeito do IG no EPOC, dificultando assim a comparação dos resultados aqui obtidos com os de outros estudos.

\section{O N C L U S Ã O}

O consumo de dietas diferindo em IG não afetou a ingestão energética durante os cinco dias consecutivos do estudo, tampouco afetou a frequência cardíaca e a pressão arterial dos voluntários durante o exercício. De maneira semelhante, os tratamentos aplicados no estudo não afetaram o gasto energético e o EPOC. O QR no período pós-prandial após o consumo de alimentos de AIG foi significantemente menor do que de BIG, tanto no primeiro quanto no quinto dia de teste. Enquanto a taxa de oxidação de carboidrato foi maior após o consumo de alimentos de BIG, a taxa de oxidação de gordura após a ingestão da refeição de AIG foi superior no período pós-prandial. Esses resultados sugerem que o consumo de refeições de BIG ricas em frutose pode não exercer efeito benéfico no controle da quantidade de gordura corporal.

Apesar da taxa de oxidação de gordura no período pós-exercício não ter diferido em função do IG da refeição consumida, constatou-se que esta foi superior no período pós-exercício do que no período pós-prandial antes do exercício em ambos os tratamentos. Este dado indica que a realização de exercício físico pode contribuir para a redução da quantidade de gordura corporal independentemente do IG da refeição consumida antes do exercício.

\section{A G RADECIMENTO}

A Fapemig (Fundação de Amparo à Pesquisa do Estado de Minas Gerais) pela concessão do auxílio financeiro (processo 1420/06) para a condução desse estudo.

\section{REFERÊ N CIAS}

1. Beraldo FC, Vaz IMF, Naves MMV. Nutrição, atividade física e obesidade: aspectos atuais e recomendações para prevenção e tratamento. Rev Med Minas Gerais. 2004; 14(1):57-62.

2. Gillette CA, Bullough RC, Melby CL. Postexercise energy expenditure in response to acute aerobic or resistive exercise. Int J Sport Nutr. 1994; 4(4): 347-60.

3. Phelain JF, Reinke E, Harris MA, Melby CL. Postexercise energy expenditure and substrate oxidation in young women resulting from exercise bouts of different intensity. J Am Coll Nutr. 1997; 16(4):140-6.

4. Yoshioka M, Doucet $E$, St-Pierre $S$, Améras $N$, Richard D, Labrie A, et al. Impact of high-intensity 
exercise on energy expenditure, lipid oxidation and body fatness. Int J Obes. 2001; 25(3): 332-9.

5. Febbraio MA, Keenan J, Angus D, Campbell S, Garnham AP. Preexercise carbohydrate ingestion, glucose kinetics, and muscle glycogen use: effect of the glycemic index. J Appl Physiol. 2000; 89(5): 1845-51.

6. Wee SL, Williams C, Tsintzas K, Boobis L. Ingestion of a high-glycemic index meal increases muscle glycogen storage at rest but augments its utilization during subsequent exercise. J Appl Physiol. 2005; 99(2):707-14.

7. Wee S, Williams C, Gray S, Horabintitle J. Influence of high and low glycemic index meals on endurance running capacity. Med Sci Sports Exerc. 1999; 31(3): 393-9.

8. Bennard P, Doucet E. Acute effects of exercise timing and breakfast meal glycemic index on exercise-induced fat oxidation. Appl Physiol Nutr Metab. 2006; 31(5):502-11.

9. Brand-Miller J, Foster-Powell K. Diets with a low glycemic index: from theory to pratice. Nutr Today. 1999; 34(2):64-72.

10. Cooper KH. O programa aeróbico para o bem estar total. Rio de Janeiro: Nórtica;1982.

11. Pollock ML, Wilmore JH. Exercício na saúde e na doença. Rio de Janeiro: Medsi; 1993.

12. Sociedade Brasileira de Hipertensão: I Diretriz Brasileira de Diagnóstico e Tratamento da Síndrome Metabólica. Rev Soc Bras Hipert 2004;7(4):1-41.

13. Foster-Powell K, Holt SHA, Brand-Miller, JC. International table of glycemic index and glycemic load values: 2002. Am J Clin Nutr. 2002; 76(1):5-56.

14. Bray GA, Gray DS. Obesity I: Phathogenesis. Western. J Med. 1988; 149(4):429-41.

15. World Health Organization. Physical Status: the use and interpretation of anthropometrics. Report of a World Health Organ Expert Committee. World Organ Tech Rep Ser. 1995; 854:1-452.

16. Mclntosh M, Miller C. A diet containing food rich in soluble and insoluble fiber improves glycemic control and reduces hyperlipidemia among patients with type 2 diabetes mellitus. Nutr Rev. 2001; 59(2): 52-5.

17. Chandalia M, Garg A, Lutjohann D, Von Bergmann K, Grundy SM, Brinkley LJ. Beneficial effects of high dietary fiber intake in patients with type 2 diabetes mellitus. New Eng J Med. 2000; 342(19):1392-8.

18. Wu CL, Nicholas C, Williams, C, Took A, Hardy L. The influence of high-carbohydrate meal with different glycaemic indices on substrate utilization during subsequent exercise. Br J Nutr. 2003; 90(6): 1049-56.

19. Food and Agriculture Organization. Carbohydrates in human nutrition. Report of an FAONHO Expert
Consultation on Carbohydrates. Rome: WHO, 1998.

20. Wolever TMS, Jenkins DJA, Jenkins AL, Josse RG. The glycemic index: methodology and clinical implications. Am J Clin Nutr. 1991; 54(5):846-54.

21. Marins J, Giannichi R. Avaliação e prescrição de atividade física. $3^{a}$ ed. Rio de Janeiro: Shape; 2003.

22. Karvonen JJ, Kentala E, Mustala O. The effects of trainning on heart rate, a "longitudinal" Study. Ann Med Esp Biol Fenn. 1957; 35(3):307.

23. Diener, JRC. Artigo de revisão: Calorimetria Indireta. Rev Assoc Med Brás. 1997; 43(3):245-53.

24. Valtueña S, Salas-Salvadó J, Lorda PG. The respiratory quotient as a prognostic factor in weight-loss rebound. Int J Obes. 1997, 21(9): 811-7.

25. Melby C, Scholl C, Edwards G, Bullough R. Effect of acute resistance exercise on post exercise energy expenditure and resting metabolic rate. J Appl Physiol 1993; 75(4):1847-53.

26. Fray KN. Calculation of substrate oxidation rates in vivo from gaseous exchange. J Appl Physiol. 1983; 55(2):628-34.

27. Abete I, Parra D, Martinez A. Energy-restricted diets based on a distinct food selection affecting the glycemic index induce different weight loss and oxidative response. Clin Nutr. 2008; 27(4):1-7.

28. Tittelbach TJ, Mattes RD, Gretebeck RJ. Postexercise substrate utilization after a high glucose vs. high fructose meal during negative energy balance in the obese. Obes Res. 2000; 8(7): 496-505.

29. Barreiros RC, Bossolan G, Trindade CEP. Fructose in humans: metabolic effects, clinical utilization, and associated inherent errors. Rev Nutr. 2005; 18 (3):377-89. doi: 10.1590/S1415-5273200500030 0010 .

30. Foureaux G, Pinto KMC, Damaso A. Efeito do consumo excessivo de oxigênio após o exercício e da taxa metabólica de repouso no gasto energético. Rev Bras Med Esporte. 2006; 12(6):393-8.

31. Matsuura C, Meirelles CM, Gomes PSC. Gasto energético e consumo de oxigênio pós-exercício contraresistência. Rev Nutr. 2006; 19(6):729-40. doi: 10.1590/S1415-52732006000600009.

32. Short KR, Sedlock DA. Excess postexercise oxygen consumption and recovery rate in trained and untrained subjects. J Appl Physiol. 1997; 83(1):153-9.

33. Almuzaini KS, Potteiger JA, Green SB. Effect of split exercise sessions on excess postexercise oxygen consumption and resting metabolic rate. Can J Apll Physiol. 1998; 23(5):433-43.

Recebido em: 24/1/2009

Versão final reapresentada em: 1/7/2010 Aprovado em: 12/8/2010 